\title{
Resource Allocation for Millimeter-Wave Train-Ground Communications in High-Speed Railway Scenarios
}

\author{
Xiangfei Zhang, Yong Niu, Member, IEEE, Shiwen Mao, Fellow, IEEE, Yunlong Cai, Senior Member, IEEE, \\ Ruisi He, Senior Member, IEEE, Bo Ai, Senior Member, IEEE, Zhangdui Zhong, Senior Member, IEEE, and \\ Yiru Liu
}

\begin{abstract}
With the development of wireless communication, higher requirements arise for train-ground wireless communications in high-speed railway (HSR) scenarios. The millimeter-wave (mm-wave) frequency band with rich spectrum resources can provide users in HSR scenarios with high performance broadband multimedia services, while the full-duplex (FD) technology has become mature. In this paper, we study train-ground communication system performance in HSR scenarios with mobile relays (MRs) mounted on rooftop of train and operating in the FD mode. We formulate a nonlinear programming problem to maximize network capacity by allocation of spectrum resources. Then, we develop a sequential quadratic programming (SQP) algorithm based on the Lagrange function to solve the bandwidth allocation optimization problem for track-side base station (BS) and MRs in this mm-wave train-ground communication system. Extensive simulation results demonstrate that the proposed SQP algorithm can effectively achieve high network capacity for trainground communication in HSR scenarios while being robust to the residual self-interference (SI).
\end{abstract}

Index Terms-Full-duplex communications, sequential quadratic programming, Train-ground communications, high-

Copyright (c) 2015 IEEE. Personal use of this material is permitted. However, permission to use this material for any other purposes must be obtained from the IEEE by sending a request to pubs-permissions@ieee.org. This study was supported by National Key R\&D Program of China (2020YFB1806903); in part by the National Natural Science Foundation of China Grants 61801016 , 61725101, 61961130391, and U1834210; in part by the State Key Laboratory of Rail Traffic Control and Safety (Contract No. RCS2021ZT009), Beijing Jiaotong University; and supported by the open research fund of National Mobile Communications Research Laboratory, Southeast University (No. 2021D09); in part by the Fundamental Research Funds for the Central Universities, China, under grant number I20JB0200030; and supported by Frontiers Science Center for Smart High-speed Railway System; in part by the State Key Laboratory of Rail Traffic Control and Safety, Beijing Jiaotong University, under Grant RCS2019ZZ005; in part by the Fundamental Research Funds for the Central Universities 2020JBM089.

X. Zhang is with the State Key Laboratory of Rail Traffic Control and Safety, Frontiers Science Center for Smart High-speed Railway System, Beijing Jiaotong University, Beijing 100044, China, (e-mail: zxfei78@ 163.com).

Y. Niu is with the State Key Laboratory of Rail Traffic Control and Safety, Beijing Jiaotong University, Beijing 100044, China, and also with the National Mobile Communications Research Laboratory, Southeast University, Nanjing 211189, China (e-mail: niuy11@163.com).

S. Mao is with the Department of Electrical and Computer Engineering, Auburn University, Auburn, AL 36849-5201, USA (e-mail: smao@ieee.org)

Y. Cai is with the Department of ISEE, Zhejiang University, China (e-mail: ylcai@zju.edu.cn).

R. He, B. Ai, Z. Zhong, and Y. Liu are with the State Key Laboratory of Rail Traffic Control and Safety, Beijing Engineering Research Center of High-speed Railway Broadband Mobile Communications, and the School of Electronic and Information Engineering, Beijing Jiaotong University, Beijing 100044, China (e-mails: ruisi.he@bjtu.edu.cn; boai@bjtu.edu.cn; zhdzhong@bjtu.edu.cn; yrliu@bjtu.edu.cn). speed railway, Millimeter-wave communications, Resource allocation.

\section{INTRODUCTION}

Looking back at the past decade, the rapid development of high-speed railway (HSR) has driven many technological innovations and changed people's lives. As of the end of April 2015, the total operating mileage of the world's HSR reached 29,700 kilometers. In particular, as of the end of 2019, the operating mileage of Chinese HSR has reached 35,000 kilometers, ranking the first in the world. Most of the Chinese HSR stations are located in the urban life circles and the highspeed trains have flexible schedules, allowing people to travel easily to effectively save costs and to improve the quality of people's lives.

On the other hand, since many passengers are accustomed to broadband wireless access in their daily living environment, more and more people hope to have high-quality broadband wireless access on mobile terminals. However, in the HSR scenarios, the high-speed of train causes frequent handovers. When the cell radius is 1 to $2 \mathrm{~km}$, the train running at $350 \mathrm{~km} / \mathrm{h}$ will handover every 10 to 20 s [1]. In addition, the rapid relative movement between the train and ground base station (BS) causes more serious Doppler shift and smaller channel coherence time. So the wireless channel in HSR scenarios has obvious non-stationary and fast time-varying characteristics, which seriously reduces the performance of train-ground communication systems [2]. Moreover, due to the complexity and non-stationarity of HSR scenarios, there are weak field strength areas and blind areas, and the train body of metal material causes great penetration loss to the signal from the BS [3]. Therefore, meeting passengers' compelling demand for broadband mobile communications in the HSR environment has become a key technical challenge. It has become particularly important to carry out research on broadband wireless communication technologies in HSR scenarios. However, the currently widely used communication technology under HSR scenarios, such as GSM-R, can only support a rate that is too low to meet user's increasing demands for multimedia applications, and the current demand rate of each train is about $37.5 \mathrm{Mbps}$. With the growth of business and entertainment activities and quality of service demands, the demand rate may easily reach $0.5-5 \mathrm{Gbps}$ in the near future [4]. Obviously, the 
current wireless transmission scheme will be inadequate to satisfy the needs of HSR passengers.

To this end, the 30-300GHz millimeter-wave (mm-wave) frequency band can provide rich spectrum resources for trainground communications in HSR scenarios. If mm-wave communications are used to carry broadband multimedia services, the transmission rate in the order of gigabit can be offered [5]. Mobile communication operators and wireless communication system designers are focusing on developing the related technologies, hoping to provide users with high-capacity broadband wireless access. Current typical application scenarios include high-speed data transmission between mobile devices, mm-wave wireless backhauls, military radar, autonomous cars, and the co-design of radar and communications, etc.

To utilize mm-wave communications for train-ground communications, high-speed data transmission can be realized with the help of mobile relays (MRs) deployed on the rooftop of the train, which can significantly improve the broadband wireless communication service performance of the entire train-ground communication system [3]. Furthermore, the fullduplex (FD) communication technology allows wireless communication devices to simultaneously transmit and receive signals on the same frequency band, and the capacity of the communication system can be doubled theoretically [6]. Therefore, it would be desirable for the MRs to adopt both the mm-wave and FD technology, and simultaneously serve HSR passengers and ground users. The track-side BS allocates part of the bandwidth to the MRs, allowing some users to directly communicate with the track-side BS in a traditional manner, and other users to be associated with the MRs to obtain wireless access. In this system model, we aim to propose an effective algorithm that can fully coordinate the ground BS and MRs to obtain maximum spectrum utilization. Due to the plentiful spectrum resources of mm-wave frequency band, and the HSR scenario has inherent high-speed timevarying characteristics, different bandwidth resource allocation methods may bring great performance differences. Therefore, determining the bandwidth allocation mechanism in the investigated train-ground communication system is a key challenge, and proposing an effective bandwidth resource allocation algorithm to maximize the network capacity is the focus of this paper.

Therefore, it is timely and relevant to carry out research on resource allocation in the train-ground communication system based on mm-wave communications and FD transmissions in HSR scenarios. Such reseach will provide theoretical and technical support for the application of mm-wave communication and FD to a new generation of HSR wireless communication systems and to improve the quality of international rail transit communication services. The current research efforts on mm-wave communication is concentrated on $28 \mathrm{GHz}, 38 \mathrm{GHz}$, $60 \mathrm{GHz}$ frequency bands and the E-band (i.e., $71-76 \mathrm{GHz}$ and $81-86 \mathrm{GHz})$. At the same time, the rapid development of Complementary Metal Oxide Semiconductor (CMOS) in radio frequency integrated circuits has paved the way for the production of mm-wave electronic devices [7]. Several international standardization efforts, such as ECMA-387 [8], IEEE 802.15.3c [9], and IEEE 802.11ad [10], focusing on indoor Wireless Personal Area Networks (WPAN) or Wireless Local Area Networks (WLAN), have made great progress.

In order to compensate for the severe link attenuation of mm-wave communications, directional antennas are used to utilize the beamforming technology for high antenna gain. Due to the short wavelength, many tiny antenna elements can be integrated in a small area. Now a variety of beam training algorithms have been proposed to help reduce the time resource required for beam training at the transmitter and receiver [11].

Due to directional transmissions, the mutual interference between mm-wave links can be significantly reduced. Mudumbai et al. [12] modeled the highly directional wireless links in outdoor mesh networks in the $60 \mathrm{GHz}$ band as "pseudo wired," i.e., the details of antenna pattern and interference between the non-adjacent links can be ignored in the MAC protocol design of the mm-wave mesh network. Son et al. [15], [16] showed that when the inter-links interference is reduced, multiple data streams can be transmitted in the same time slot to achieve maximizing the space division multiplexing gain. On the other hand, the directional transmissions also make it hard to implement the carrier sense method used by broadcast channels to avoid collision, which is called the "deafness" problem [15]. Therefore, the design of the MAC protocol in mm-wave communication scenarios needs to consider the coordination mechanism of mm-wave links and make full use of concurrent transmissions to increase the network capacity.

On the other hand, as a technology with the great potential to increase the wireless capacity, the FD communication is regarded as one of the key physical layer technologies of the 5th generation mobile networks $(5 \mathrm{G})$, which has triggered great attention in academia and industry in recent years [17]. However, when the FD technology is adopted, the signal at the transmitting end leaks to the corresponding receiving end to cause strong self-interference (SI). At present, the biggest challenge in applying FD technology in different scenarios is to eliminate such SI. In order to improve the spectrum efficiency of the FD communication, a variety of physical layer techniques such as antenna interference cancellation, radio frequency interference cancellation and digital interference cancellation have been investigated. Cui et al. [18] proposed an optimal MR selection scheme in the FD multi-relay communication scenarios to maximize the signal-to-interference plus noise ratio. In order to cope with the SI in the FD MultipleInput Multiple-Output (MIMO) relay network, Rahman et al. [19] proposed an efficient SI elimination algorithm based on the Space Projection Algorithm (SPA). With the latest techniques, the SI level can be reduced to $-110 \mathrm{~dB}$, ensuring that the FD technology can be applied in practical scenarios.

However, there has been very limited prior work on applying these two advanced technologies, i.e., mm-wave communications and FD, to train-ground communication system in HSR scenarios. In this paper, we study the bandwidth resource allocation mechanism for the mm-wave train-ground communication system where the MRs adopt FD communications. We focus on the coordination of bandwidth resource allocation between track-side BS and FD MRs in the HSR scenario, aiming at network capacity optimization for the train- 
ground communication system. It is foreseeable that if $\mathrm{mm}$ wave communication and FD can be adopted in train-ground communication scenarios, and the bandwidth allocation mechanism developed in this paper will be useful for achieving high global network data transmission rates. The main contributions made in this paper are summarized as follows.

- We introduce FD technique to MRs deployed on rooftop of train cabin in mmWave train-ground communication systems. Background noise and residual SI (RSI) are simultaneously taken into account so that the advantages of the FD and mmWave can be fully utilized.

- By taking the bandwidth allocation ratio of track-side BS and FD MRs as the variable, we formulate the bandwidth allocation problem in the established mmWave train-ground communication systems as a nonlinear programming problem. Then, we take maximizing network capacity as the goal, and propose a fast-converging bandwidth allocation algorithm which can ensure high network capacity and a certain anti-SI ability.

- We evaluate the proposed algorithm in the $60 \mathrm{GHz}$ mmWave train-ground communication systems with limited spectrum resource. The extensive simulations demonstrate that compared with other traditional priority algorithms and optimization algorithms, the proposed SQP algorithm based on Lagrangian function can significantly increase the network capacity and make the mmWave train-ground communication systems have a certain antiSI ability. Furthermore, we also give insights into the reasons for the formation of some system performance.

The rest of the paper is organized as follows. In Section III. we provide an detailed overview of the related work. In Section III. we introduce the train-ground communication system model, and formulate the problem of bandwidth allocation between half-duplex (HD) track-side BS and FD MRs. The proposed SQP algorithm is presented in Section IV and evaluated in Section V] Finally, Section VI concludes this paper.

\section{RELATED WORK}

We can classify the related works into three categories: (a) mm-wave communications in wireless networks; (b) FD communications in wireless networks; and (c) application of optimization theory into resource allocation. We examine these related work in this section.

a) Mm-wave Communication: There has been considerable research on mm-wave based wireless communications and networks. Mm-wave has the advantage of large bandwidth, and the short coverage range also makes it a good application in heterogeneous networks. Niu et al. [20] summarized the characteristics of mm-wave communications, the research status in the physical layer and MAC layer, and the existing problems and challenges, and discussed the future research directions. Elkashlan et al. [5] showed that the mm-wave frequency band can provide users with high-capacity wireless broadband access, e.g., a multi-gigabit transmission rate for broadband multimedia services. Niu et al. [21] developed an energy-efficient scheme to jointly optimize power control and concurrent transmission scheduling of mm-wave backhauling in small cells and densely deployed heterogeneous cellular networks (HCNs). A blind neighbor discovery algorithm with bounded discovery time and guaranteed discovery performance was proposed in [39], while a link scheduling algorithm was developed in [40] for mm-wave ad hoc networks under blockage and interference conditions. However, compared to sub-6GHz communication systems, mm-wave communications suffer considerably greater propagation loss. In practical applications, directional antennas are often used to achieve beamforming to increase antenna gain. In the established mm-wave train-ground communication system model, many directional antennas are employed.

At present, the research of mm-wave in HSR scenarios has also made progress. Yang et al. [41] and He et al. [42] measured the mm-wave propagation in HSR scenarios, and accordingly studied the path loss characteristics. Based on the path loss measurement at $90 \mathrm{GHz}$ in a railway viaduct scenario, similar calibration works are also performed in [43]. Gao et al. [44] proposed a strategy that can solve the edge caching and content delivery problem for both HSR passengers and low-mobility cellular users. Wang et al. [23] proposed an energy-efficient power-control scheme for train-ground mmwave communications in HSR scenarios. However, due to the difficult propagation characteristics of mm-wave and the particularity of HSR scenarios, there are still many challenges ahead.

b) FD Communications: FD wireless communications have also attracted much efforts in the research community in the past decade. For example, Duarte et al. [45] designed a multi-antenna FD physical layer structure and an FD MAC layer structure compatible with the existing 802.11 standard. It turns out that the FD communication mode has the potential to nearly doubled the system throughput, and the applications of the FD mode in future WiFi standards will have potentially huge benefits. The energy-delay trade-off in a multi-channel FD wireless LAN was derived in [46] with an application of Lyapunov optimization, while a distributed power control algorithm was developed in [47] for FD wireless networks by applying the dual-decomposition approach. Feng et al. [48] showed that the gain of FD depends on the network topology and other settings; its not always the case that FD is better than HD. A joint duplex mode selection, channel allocation, and power control scheme was developed for FD HCNs. Wen et al. [24] proposed a resource allocation algorithm in a time-division multiplexed cellular network to maximize the throughput of the system while meeting the quality of service requirements of each user as much as possible. In this system, the users' equipments use the traditional HD communication mode, while the BS uses the FD communication mode.

Xiao et al. [49] explored the proper antenna configuration for FD mm-wave communications, and established a related model of the mm-wave SI channel. Ding et al. [25] proposed a QoS-aware FD concurrent transmission algorithm to maximize the number of data streams that meet user QoS requirements. Skouroumounis et al. [26] evaluated the impact of the FD mode in mm-wave communications, and proposed an analytical framework based on stochastic geometry to evaluate 
heterogeneous FD mm-wave cellular network performance from both cell center users and cell edge users. Anokye et al. [50] established a kind of cellular network topology, where the large-scale multiple-input multiple-output (MIMO) system is applied in the backhaul to enable FD communications. At the same time, according to the actual number of antennas required and the formed SI thereby, a wireless communication system with both HD and FD communications was proposed. Wang et al. [27] studied the optimal allocation of sub-channels under dense deployment of micro-cells in mm-wave networks, and proposed a sub-channel allocation method based on alliance game theory, which improved resource utilization and system throughput.

The related work show that FD communications have great research value in cellular networks, WiFi networks, mmwave networks, and heterogeneous networks. However, there are still few applications of FD in HSR scenarios. In the train-ground communication system model established in this paper, we exploit the advantages of FD technology and mmwave communications, which can jointly bring about huge improvements on spectrum efficiency and network capacity.

c) Optimization Resource Allocation: Optimization theory has been widely applied in wireless networks to improve the utilization of limited resources, such as spectrum and power. For instance, Chen et al. [22] proposed a coalition formation game to optimize resource allocation to maximize the system sum rate of HCNs in the statistical average sense. Liu et al. [29] studied the sub-channel allocation problem in two-tier OFDMA femtocell networks, and proposed a resource optimization method based on ant colony optimization to maximize the rate of multiple femtocells systems, while considering the cross-tier interference between the macrocell and multiple femtocells. Xiao et al. [28] used robust optimization theory to model uncertain interference channels and considered channel estimation errors to maximize the network throughput while avoiding the severe inter-tier interference. With the Lagrangian dual method, the original optimization problem was decomposed into an original problem and a dual problem to be solved.

Motivated by these related works, we explore optimization theory to model the bandwidth allocation problem of BS and MRs in the train-ground communication system. The key is to introduce a suitable algorithm to maximize the network capacity. The proposed algorithm is designed to solve the bandwidth allocation problem. It is also hoped that it has a certain degree of anti-SI ability for train-ground communication applications.

\section{SyStem OVERVIEW AND PROBlem Formulation}

\section{A. System Model}

This paper considers an mm-wave train-ground communication system using FD MRs, as shown in Fig. 11. In this model, the track-side BS reserves part of the bandwidth, and the remaining bandwidth is allocated to the MRs for FD communications. In addition, there are multiple MRs operating in the FD mode deployed on the train. The bandwidth resources allocated to each MR are mutually exclusive. All the devices in this train-ground communication system work in the mmwave frequency band. Both the BS and the MRs are equipped

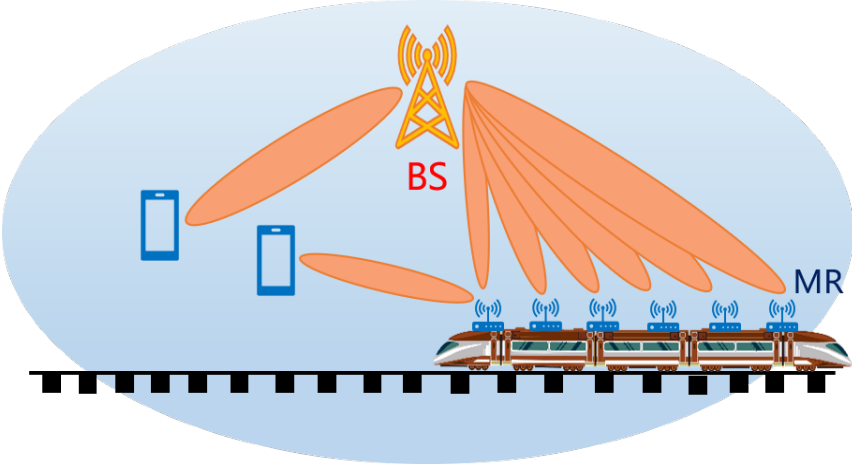

Fig. 1. Illustration of an mm-wave train-ground communication system using multiple FD MRs.

with steerable directional antennas, which allow the BS and the MRs to aim at their associated users to achieve higher antenna gain.

The track-side BS and each MR are associated with different users, and it is assumed that these association relationships have been determined in advance. Assume that multiple served users access the BS or a specific MR with Time Division Multiplexing Access (TDMA), and the time slot resources are equally allocated. In the following, for this mm-wave trainground communication system using FD MRs, it is considered that the total initial system bandwidth, i.e., the sum of the bandwidth of the BS and the bandwidth allocated to all MRs, is constant. We hope to find the best allocation ratio of bandwidth resources to achieve high network capacity. When the train is stationary, the global network capacity of this system is defined as the sum of the average user rates at the BS and that at all the MRs.

Since the train-ground communication network works in the mm-wave frequency band, non-line-of-sight transmissions will cause greater attenuation. Considering that both the BS and the MRs are equipped with steerable directional antennas, this paper assumes that all the nodes in the system use the line-ofsight links for transmission. According to the path loss model in [30], the received power at receiver $r_{k}$ from its associated server transmitter $t_{s}$ can be expressed as

$$
P_{r}(s, k)=k_{0} \cdot G_{t}(s, k) \cdot G_{r}(s, k) \cdot l_{s \rightarrow k}^{-n} \cdot P_{t},
$$

where $k_{0}$ is a constant coefficient, which is proportional to $\left(\frac{\lambda}{4 \pi}\right)^{2}, \lambda$ is the wavelength of the carrier frequency, $n$ is the path loss index, $P_{t}$ represents the transmit power of the transmitter $t_{s}, G_{t}(s, k)$ is the transmit antenna gain of transmitter $t_{s}$ in the direction of user $k$ 's receiver $r_{k}, G_{r}(s, k)$ represents the receive antenna gain from the server transmitter $t_{s}$ to the user $k$ 's receiver $r_{k}, l_{s \rightarrow k}$ indicates the distance between user $k$ 's receiver $r_{k}$ and the server transmitter $t_{s}$.

Due to the inherent multipath effect of wireless channels, the mm-wave channel is modeled as a Gaussian channel. According to the Shannon channel capacity formula, The data transmission rate $R_{s}(k)$ obtained by user $k$, who accesses the 
network through service device $s$, can be expressed as

$$
R_{s}(k)=\eta \cdot W_{s} \cdot \log _{2}\left(1+\frac{P_{r}(s, k)}{N_{0} W_{s}}\right),
$$

where $\eta \in(0,1)$ characterizes the efficiency of the signal transceiver, $W_{s}$ represents the bandwidth resource reserved for the service device $s$ according to the bandwidth allocation decision, and $N_{0}$ is the unilateral noise power spectral density of the Gaussian channel.

\section{B. Problem Formulation}

Let the total system bandwidth be $W$ and the bandwidth reserved by the track-side $\mathrm{BS}$ be denoted by $W_{B S}$. We introduce a bandwidth resource allocation factor vector $\alpha$, the length of which is the sum of the numbers of MRs and BS in the system. The following formulation is based on the scenario of a single BS and multiple MRs, which, however, can be easily extended to the more general cases. The first element $\alpha_{0}$ of $\boldsymbol{\alpha}$ is the ratio of the bandwidth resources allocated to the BS, i.e., $W_{B S}$, over the total bandwidth $W$. The remaining elements $\alpha_{i}$ represents the ratio of the bandwidth allocated to MR $i$, i.e., $W_{i}$, over $W$. The related mathematical relations of bandwidth resources in the system can be expressed as in (3).

$$
\left\{\begin{array}{l}
W=W_{B S}+\sum_{i=1}^{R n u m} W_{i} \\
W_{B S}=W \cdot \alpha_{0} \\
W_{i}=W \cdot \alpha_{i}, \quad i=1,2, \ldots, R_{\text {num }}
\end{array}\right.
$$

where Rnum represents the total number of MRs in the HSR scenario.

The total number of users in the train-ground communication system is denoted as $M$, the number of users associated with the $\mathrm{BS}$ is denoted as $M_{B S}$, and the number of users associated with MR $i$ is denoted as $M_{i}$. Using the received power model (1), the received power by user $k$ associated with the BS (with transmit power $P_{t}(B S)$ ) can be expressed as

$$
P_{r}(B S, k)=k_{0} \cdot G_{t}(B S, k) \cdot G_{r}(B S, k) \cdot l_{B S \rightarrow k}^{-n} \cdot P_{t}(B S) \text {. }
$$

Similarly, for the $M_{i}$ users associated with MR $i$, the received power by user $j$ from MR $i$ (with transmit power $P_{t}(i)$ ) can be expressed as

$$
P_{r}(i, j)=k_{0} \cdot G_{t}(i, j) \cdot G_{r}(i, j) \cdot l_{i \rightarrow j}^{-n} \cdot P_{t}(i) .
$$

The data transmission rate of user $k$ associated with the BS is denoted as $R_{B S}(k)$, which can be expressed as

$$
R_{B S}(k)=\eta \cdot W_{B S} \cdot \log _{2}\left(1+\frac{P_{r}(B S, k)}{N_{0} W_{B S}}\right) .
$$

Since the BS uses TDMA for associated users, the average transmission rate of the users associated with the BS can be expressed as

$$
\bar{R}_{B S}=\frac{1}{M_{B S}} \sum_{k=1}^{M_{B S}} R_{B S}(k) .
$$

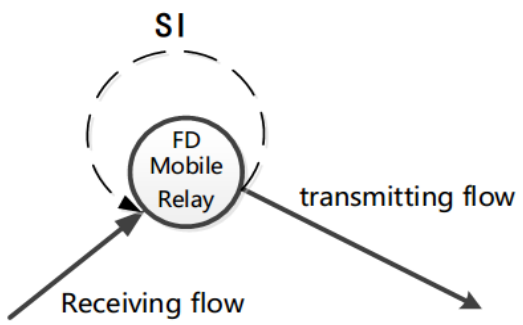

Fig. 2. SI at an FD MR.

Similarly, the rate of user $j$ associated with MR $i$ is denoted as $R_{i}(j)$, which can be expressed as

$$
R_{i}(j)=\eta \cdot W_{i} \cdot \log _{2}\left(1+\frac{P_{r}(i, j)}{N_{0} W_{i}+I_{s}(i)}\right) .
$$

It should be noted that, because the MRs operates in the FD mode, the signal from a certain MR will suffer from the background noise as well as a certain amount of SI (i.e., due to imperfect SI cancellation). As shown in Fig. 2, the so-called SI refers to the interference at the MR receiver caused by its own transmitter. We introduce

$$
I_{s}(i)=\beta \cdot P_{t}(i),
$$

to model the residual SI level at $\mathrm{MR} i$, where $\beta$ is the SI cancellation level. The smaller the $\beta$, the more effective the SI cancellation at the MR. Since the MRs also use TDMA for the data transmissions of their associated users, the average transmission rate of the users associated with MR $i$ can be expressed as

$$
\bar{R}_{i}=\frac{1}{M_{i}} \sum_{j=1}^{M_{i}} R_{i}(j)
$$

According to the train-ground communication system model, we next formulate the mathematical model for the goal of maximizing network capacity. With the models given in Section III-A and this section, the objective function of the bandwidth allocation problem can be expressed as

$$
\max \bar{R}_{B S}+\sum_{i=1}^{\text {Rnum }} \bar{R}_{i}
$$

where $\bar{R}_{B S}$ is the average data transmission rate of the users associated with the $\mathrm{BS}$, and $\bar{R}_{i}$ represents the average data transmission rate of the users associated with MR $i$.

Aiming at maximizing the global network capacity of the train-ground communication system, we have established a mathematical model that takes multi-dimensional bandwidth allocation factor $\boldsymbol{\alpha}$ as a variable. The objective function is a multivariate nonlinear function. The constraints on the bandwidth allocation factor $\boldsymbol{\alpha}$ in practical HSR scenarios can be expressed as

$$
\begin{aligned}
& \alpha_{0}+\sum_{i=1}^{\text {Rnum }} \alpha_{i}=1 \\
& 0 \leq \alpha_{i} \leq 1, \quad i=0,1, \ldots, \text { Rnum } .
\end{aligned}
$$




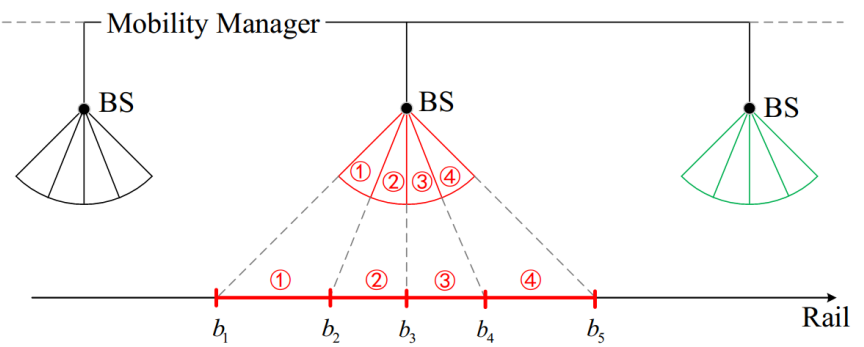

Fig. 3. Beam switching to get a LOS link.

Due to the short wavelength, mm-wave is vulnerable to various blockages, such as foliage, buildings, and viaducts in railway environments. The link blockage occurs when obstacles appear in the radio links between the transceivers, which results in received signal strength degradation caused by severe attenuation [31]. When the achieved signal to interference plus noise ratio (SINR) at the receiver (RX) side is lower than the required threshold $\gamma_{t h}$, the system is unable to guarantee the required bit error rate and the link is considered to be turned off [32]. The link blockage depends on multiple factors, including the surrounding environment, obstacle density, beamwidth, and transmission distance.

Considering the height of the MRs, the link between the MRs and the ground BS can be regarded as line-of-sight (LOS) transmission, and the railway is generally located in spacious scenarios, so it is considered that the blockages are mainly caused by human body. We consider that in the investigated mm-wave train-ground communication system, the blockage problem caused by human body can be solved in three steps. In the first step, we connect the users to the nearest $\mathrm{BS}$ or MR, and propose an average outage probability $P_{b}$ for all links, which note that each link has a random probability of experiencing blockage. In the second step, if the communication link between the user and the associated equipment experiences blockage, this user will re-establish an association relationship with the second closest equipment to actively avoid blockage. In the third step, we study the scenario of non-LOS (NLOS) transmission, which can be enhanced with the assist of intelligent reflecting surface (IRS) and Device-to-Device (D2D) communications. In this paper, for analytical simplicity, it is assumed that the link blockage probability remains stable in a road segment. The link blockage probability $P_{b}$ on average is deemed as a constant in a certain section along the rail track [33].

In order to construct a LOS link in the investigated mm-wave train-ground wireless communications, we consider adopting the beam switching for beam alignment. The $\mathrm{mm}$ wave BS communicates with the MRs using directional beamforming at the TX and RX. Train control systems (TCSs) are used in modern railway systems, which can help us obtain the train position and speed information in time and achieve efficient beam alignment. Each beam can be projected on to the rail as shown in Fig. 33, which defines its coverage [34]. If it is known that MR have entered the coverage of a certain beam, then this beam is the best choice of the MR for mm-wave

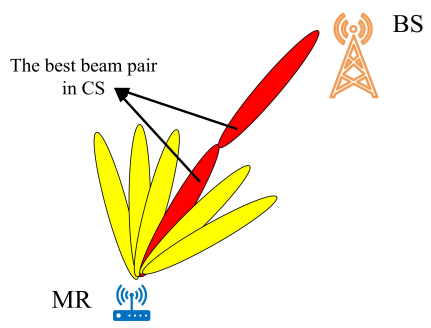

(a)

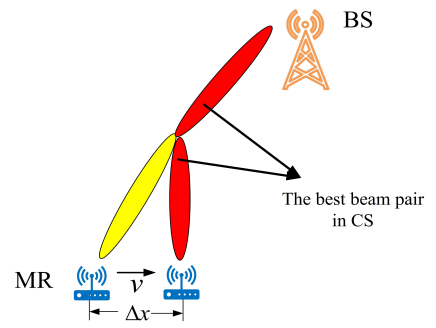

(b)
Fig. 4. The IA process: (a) CS phase, (b) RA phase.

communications with BS. Since the speed of the high-speed train is relatively stable during the driving process, this beam alignment approach using train position information avoids the cumbersome traditional beam training process [35].

In current mm-wave systems, the entire initial access (IA) process consists of two parts, the cell search (CS) and random access (RA) phases [36] [37], and beam switching avoids waste caused by beam scanning. As shown in Fig. 4(a), in the CS phase, MRs measure the signal quality of each beam pair according to all Feasible direction in the beam coverage area, and record the index of the best transmitting beam. In the following RA phase as shown in Fig. 4(b), at first users randomly select a preamble from a predefined preamble set in which the preambles are mutually orthogonal [38]. Usually, the number of orthogonal preambles determines the maximum number of MRs to be allowed to simultaneously access this network. By coding the recorded index of the best transmitting beam with the selected preamble, the user then sends this information through the exact receiving direction from which the best transmitting beam is detected during the previous CS phase. Meanwhile, the BS can capture this feedback [35].

Besides, in mm-wave systems, the preamble-coded information of the RA phase is carried by narrow beams and transmitted only to the direction where the best transmitting beam is detected in the CS phase, thereby highly decreasing the access collision probability compared with omni-directional wireless systems [36].

In summary, the problem of optimal bandwidth allocation, denoted by P1, can be formulated as follows.

$$
\text { (P1) } \max \left(1-P_{b}\right)\left(\bar{R}_{B S}+\sum_{i=1}^{\text {Rnum }} \bar{R}_{i}\right)
$$

s.t. Constraints (12) and (13).

Because the data transmission rate of each user includes a logarithmic function, the objective function is nonlinear. So problem (P1) is a nonlinear programming problem. In the next section, we introduce a solution algorithm to solve problem (P1) with competitive solutions.

\section{BANDWidth Allocation Algorithm}

In this section, we will introduce a sequential quadratic programming (SQP) algorithm based on Lagrangian function for solving (P1). With the help of the Lagrangian function, the proposed algorithm ensures that the approximated subproblem of the original problem is always a convex optimization problem, and the SQP algorithm ensures the speed of 
convergence to the optimal solution. In the following, we first describe how to construct the Lagrangian function, and then present the SQP algorithm in detail.

\section{A. Lagrange Function}

The traditional Lagrangian multiplier method first constructs a Lagrangian function, and then obtains the partial derivative with respect to each variable and the Lagrange multipliers. Letting the partial derivatives be equal to 0 , finally the extreme values of the problem can be obtained. In problem (P1), according to the constraint conditions for the bandwidth allocation factor $\alpha$ in (12) and (13), it can be seen that these contains include an equality condition and several inequality conditions. According to [14], the Lagrangian multiplier method can effectively solve the equality constraint problem and the inequality constraint problem.

The Lagrangian function of problem (P1) is given by

$$
\begin{aligned}
\mathcal{L}(\boldsymbol{\alpha}, \mu, \gamma)= & \bar{R}_{B S}+\sum_{i=1}^{\text {Rnum }} \bar{R}_{i}+\mu\left(\alpha_{0}+\sum_{i=1}^{\text {Rnum }} \alpha_{i}-1\right) \\
& +\sum_{j=0}^{2(\text { Rnum }+1)} \sum_{i=1}^{\text {Rnum }} \gamma_{j} \cdot g_{j}\left(\alpha_{i}\right)
\end{aligned}
$$

where $\mu$ and $\gamma$ are Lagrange multipliers, the sum of the first two items on the right side is the objective function of problem (P1), i.e., the network capacity. The third term takes into account the equality constraint in $(12)$. The fourth term takes into account the inequality constraints in (13), the specific form is to write the constraints $\alpha_{i} \in(0,1), i=0,1, \ldots, R_{\text {num }}$ in (13) as two inequalities, which must be in accordance with the form $g_{j}\left(\alpha_{j}\right) \leq 0$. Take the bandwidth allocation factor $\alpha_{0}$ of the BS as an example, the two inequality constraints can be expressed as

$$
\left\{\begin{array}{l}
g_{0}\left(\alpha_{0}\right)=\alpha_{0}-1 \leq 0 \\
g_{1}\left(\alpha_{0}\right)=-\alpha_{0} \leq 0
\end{array}\right.
$$

Now, the actual conditions $0 \leq \alpha_{i} \leq 1$ that each bandwidth allocation factor needs to meet can be obtained as in (16).

In addition, the feasible solution obtained by using the Lagrangian function involving both equality and inequality constraints needs to meet the Karush-Kuhn-Tucker (KKT) condition [14]. For problem (P1), the KKT condition of the corresponding Lagrangian function can be expressed as

$$
\left\{\begin{array}{l}
\nabla_{\boldsymbol{\alpha}} \mathcal{L}(\boldsymbol{\alpha}, \mu, \boldsymbol{\gamma})=0 \\
\gamma_{j} \cdot g_{j}\left(\alpha_{j}\right)=0, \quad j=0,1, \ldots, 2(\text { Rnum }+1) \\
g_{j}\left(\alpha_{j}\right) \leq 0, \quad j=0,1, \ldots, 2(\text { Rnum }+1) \\
\alpha_{0}+\sum_{i=1}^{\text {Rnum }} \alpha_{i}-1=0 \\
\gamma_{j} \geq 0, \quad j=0,1, \ldots, 2(\text { Rnum }+1) .
\end{array}\right.
$$

In (17), the first equation represents the use of the constructed Lagrangian function to obtain the partial derivative with respect to the bandwidth allocation variable, which is also a necessary condition for the Lagrangian multiplier method to obtain a feasible solution. The second equation is the Lagrangian slack complementary condition, while the third and fourth lines are the initial constraints that need to be met in problem (P1). And the fifth line is the extension under the existence of inequality constraints. These are the conditions that need to be met by the feasible solution obtained by the Lagrangian multiplier method. Each solution obtained by the Lagrange multiplier method will be judged, and the solution satisfying the KKT conditions is the optimal solution to the original optimization problem.

It can be shown that the solution that satisfies the KKT conditions, obtained by constructing a Lagrangian function, is also a feasible solution to problem (P1). Before verification, recall the mm-wave train-ground communication system model using the FD MRs. Assuming that there are one track-side BS and nine MRs in the system model, then the dimension of the bandwidth allocation vector $\boldsymbol{\alpha}$ is 10, and the objective function is a non-linear function of 10 variables. The overall constraints include one equation and 20 inequalities, and then the partial derivatives of the Lagrangian function contain 31 variables. Solving the system of nonlinear equations will be highly complicated when the system is large. Therefore, we consider using the optimization algorithm to perform multiple iterations to quickly approximate the optimal solution. Dynamic adjustment of the convergence accuracy can also increase the calculation speed or accuracy, and it provides greater flexibility for the real communication scenarios to deal with real-time service requirements.

\section{B. SQP Algorithm}

The SQP method transforms the originally complicated nonlinear programming problem into a series of quadratic programming sub-problems. In the beginning, an approximation solution is used to approximate the objective function of the original problem, which is simplified into a quadratic programming sub-problem. And the approximation solution of the original problem is obtained with this quadratic programming sub-problem. If the convergence accuracy is met, the obtained approximation solution is considered to be the optimal solution to the original problem. Otherwise, the starting point is selected again to repeat the above procedure. This is an iterative calculation process to solve the complicated nonlinear programming problem.

Compared with other optimization algorithms, SQP has the outstanding advantages of fast convergence, high computational efficiency, and strong boundary search ability, and has been widely used. On the other hand, in order to achieve global convergence, it is usually required that the second derivative matrix $H$ of the objective function of the quadratic programming sub-problem is symmetric and positive definite. At this point, this sub-problem can be further characterized as a strict convex quadratic programming problem. This type of problems have a unique solution, i.e., the local optimal solution is also the global optimal solution, and it is relatively easy to obtain this unique solution. Moreover, the positive definiteness of matrix $H$ ensures that the search direction obtained by solving the quadratic programming sub-problem is indeed the decreasing direction of the objective function value of the original problem. The specific implementation principle of the SQP algorithm is given below. 
Consider a nonlinear programming problem that includes both equality and inequality constraints given by (18).

$$
\begin{aligned}
\min & f(\mathbf{X}) \\
\text { s.t. } & g_{u}(\mathbf{X}) \leq 0, \quad u=1,2, \ldots, p \\
& h_{v}(\mathbf{X})=0, \quad v=1,2, \ldots, m,
\end{aligned}
$$

Obviously, where $\mathbf{X}$ represents multi-dimensional variables. Then, applying Taylor series expansion, the objective function of the original nonlinear programming problem can be approximated by the first- and second-order terms (i.e., by discarding higher-order terms) at the iteration point $\mathbf{X}_{k}$, while the constraint functions are approximated by their first-order terms. This way, the original problem is simplified into a quadratic programming sub-problem as given in (19).

$$
\begin{gathered}
\min f(\mathbf{X})=\frac{1}{2}\left[\mathbf{X}-\mathbf{X}_{k}\right]^{\mathrm{T}} \nabla^{2} f\left(\mathbf{X}_{k}\right)\left[\mathbf{X}-\mathbf{X}_{k}\right] \\
+\nabla f\left(\mathbf{X}_{k}\right)^{\mathrm{T}}\left[\mathbf{X}-\mathbf{X}_{k}\right] \\
\text { s.t. } \nabla g_{u}\left(\mathbf{X}_{k}\right)^{\mathrm{T}}\left[\mathbf{X}-\mathbf{X}_{k}\right]+g_{u}\left(\mathbf{X}_{k}\right) \leq 0, \quad u=1,2, \ldots p \\
\quad \nabla h_{v}\left(\mathbf{X}_{k}\right)^{\mathrm{T}}\left[\mathbf{X}-\mathbf{X}_{k}\right]+h_{v}\left(\mathbf{X}_{k}\right)=0, \quad v=1,2, \ldots, m .
\end{gathered}
$$

It is worth noting that the constrained optimization problem (19) is an approximation of the original problem (18), but its solution is not necessarily a feasible solution to the original problem. Therefore, we define a new problem variable $\mathbf{S}$, and its mathematical relationship with the original variable $\mathbf{X}$ can be expressed as

$$
\mathbf{S}=\mathbf{X}-\mathbf{X}_{k}
$$

With 20, , we can rewrite the simplified quadratic programming sub-problem as a nonlinear programming problem with variable $\mathbf{S}$, given by

$$
\begin{array}{ll}
\min & f(\mathbf{S})=\frac{1}{2} \mathbf{S}^{\mathrm{T}} \nabla^{2} f\left(\mathbf{X}_{k}\right) \mathbf{S}+\nabla f\left(\mathbf{X}_{k}\right)^{\mathrm{T}} \mathbf{S} \\
\text { s.t. } & \nabla g_{u}\left(\mathbf{X}_{k}\right)^{\mathrm{T}} \mathbf{S}+g_{u}\left(\mathbf{X}_{k}\right) \leq 0, u=1,2, \ldots, p \\
& \nabla h_{v}\left(\mathbf{X}_{k}\right)^{\mathrm{T}} \mathbf{S}+h_{v}\left(\mathbf{X}_{k}\right)=0, v=1,2, \ldots, m .
\end{array}
$$

We also define the following for 21).

$$
\left\{\begin{array}{l}
\mathbf{H}=\nabla^{2} f\left(\mathbf{X}_{k}\right) \\
\mathbf{C}=\nabla f\left(\mathbf{X}_{k}\right) \\
\mathbf{A}=\left[\nabla g_{1}\left(\mathbf{X}_{k}\right), \nabla g_{2}\left(\mathbf{X}_{k}\right), \ldots, \nabla g_{p}\left(\mathbf{X}_{k}\right)\right]^{\mathrm{T}} \\
\mathbf{A}_{e q}=\left[\nabla h_{1}\left(\mathbf{X}_{k}\right), \nabla h_{2}\left(\mathbf{X}_{k}\right), \ldots, \nabla h_{m}\left(\mathbf{X}_{k}\right)\right]^{\mathrm{T}} \\
\mathbf{B}=\left[g_{1}\left(\mathbf{X}_{k}\right), g_{2}\left(\mathbf{X}_{k}\right), \ldots, g_{p}\left(\mathbf{X}_{k}\right)\right]^{\mathrm{T}} \\
\mathbf{B}_{e q}=\left[h_{1}\left(\mathbf{X}_{k}\right), h_{2}\left(\mathbf{X}_{k}\right), \ldots, h_{m}\left(\mathbf{X}_{k}\right)\right]^{\mathrm{T}}
\end{array}\right.
$$

This way, the general form of the quadratic programming problem is formed, which is given by

$$
\begin{aligned}
\min & \frac{1}{2} \mathbf{S}^{\mathrm{T}} \mathbf{H S}+\mathbf{C}^{\mathrm{T}} \mathbf{S} \\
\text { s.t. } & \mathbf{A} \mathbf{S} \leq \mathbf{B} \\
& \mathbf{A}_{e q} \mathbf{S}=\mathbf{B}_{e q} .
\end{aligned}
$$

To solve the quadratic programming sub-problem (23), the optimal solution $\mathbf{S}^{*}$ is taken as the next search direction of the original optimization problem, and a constrained onedimensional search will be performed on the original optimization problem in this search direction. The one-dimensional search mentioned here is also called linear search, which refers to the optimization problems of single-variable function, and it is the basis of multi-variable function optimization problems. The common dichotomy and interpolation are typical onedimensional search algorithms. The solution obtained based on the constrained one-dimensional search can be regarded as an approximation solution $\mathbf{X}_{k+1}$ of the original problem. Iterative calculation of the above process can obtain the optimal solution to the original problem within the prescribed convergence accuracy. The key of implementing the SQP method based on the above ideas is how to quickly calculate the second derivative matrix $\mathbf{H}$ of the objective function.

Mathematicians Polomares and Mangasarian proposed a calculation method of $\mathbf{H}$ in 1976, using the Hessian matrix of the Lagrangian function constructed based on the original optimization problem, to achieve continuous correction of the second derivative matrix $\mathbf{H}$ of the objective function of the original problem [51]. The Hessian matrix here refers to a matrix composed of the arrangement of the second-order partial derivatives of a multivariate function. Simply put, each iteration performed by the SQP will make the matrix $\mathbf{H}$ of the quadratic programming sub-problem closer to the secondorder derivative matrix of the objective function of the original problem. The specific iterative updates to $\mathbf{H}$ is given by

$$
\begin{aligned}
& \mathbf{H}_{k+1}=\mathbf{H}_{k}+\frac{\mathbf{q}_{k} \mathbf{q}_{k}^{\mathrm{T}}}{\mathbf{q}_{k}^{\mathrm{T}} \mathbf{S}_{k}}-\frac{\mathbf{H}_{k}^{\mathrm{T}} \mathbf{H}_{k}}{\mathbf{S}_{k}^{\mathrm{T}} \mathbf{H}_{k} \mathbf{S}_{k}} \\
& \mathbf{S}_{k}=\mathbf{X}-\mathbf{X}_{k} \\
& \mathbf{q}_{k}=\nabla f\left(\mathbf{X}_{k+1}\right)+\sum_{u=1}^{p} \gamma_{u} \nabla g_{u}\left(\mathbf{X}_{k+1}\right)+\sum_{v=1}^{m} \mu_{v} \nabla h_{v}\left(\mathbf{X}_{k+1}\right) \\
& -\left[\nabla f\left(\mathbf{X}_{k}\right)+\sum_{u=1}^{p} \gamma_{u} \nabla g_{u}\left(\mathbf{X}_{k}\right)+\sum_{v=1}^{m} \mu_{v} \nabla h_{v}\left(\mathbf{X}_{k}\right)\right]
\end{aligned}
$$

In the iterative process of the SQP algorithm, as long as it is guaranteed that $\mathbf{q}_{k}^{\mathrm{T}} \mathbf{S}_{k}$ is positive and $\mathbf{H}$ is initialized to a positive definite matrix, the Hessian matrix will always remain positive definite. 24 is called BFGS correction method. Therefore, the key problem of applying the SQP algorithm to solve problem (P1) has been solved.

Sorting out the basic ideas of the SQP algorithm, combined with problem (P1) studied in this paper, we find that the objective function of problem (P1) is the function each time the SQP algorithm performs a secondary approximation, and the so-called "secondary approximation" is actually a secondorder Taylor expansion of the objective function, but each iteration is carried out at a different operating point. Section IV-A describes the process of constructing a Lagrangian function for problem (P1), and this Lagrangian function is used to modify the positive definite matrix $\mathbf{H}$, which is needed every time the SQP algorithm simplifies the original complicated nonlinear programming problem. Quadratic programming is a natural transition from linear programming to nonlinear programming. Finally, by repeatedly determining the search direction and performing iterative calculations, the optimal solution to problem (P1) can be obtained when the convergence accuracy condition is satisfied. 
The pseudo code of the SQP algorithm is given in Algorithm 1. which is executed at the centralized controller (CC) located in the backbone network. It can be seen from Algorithm 1 that the Lagrangian function is constructed by sorting out the objective function and the related constraints of the original bandwidth allocation problem. Then the SQP algorithm is used to simplify the complicated nonlinear programming problem to a series of quadratic programming subproblems. In the iterative calculation process, the constructed Lagrangian function is used to modify the second derivative matrix $\mathbf{H}$ of the original objective function. By solving the quadratic programming sub-problems, judging the convergence accuracy, and iterative calculating, the approximation solution to the original problem (P1) is obtained.

The computational complexity analysis of the proposed Algorithm 1 is crucial and necessary. Algorithm 1 solves a quadratic programming sub-problem obtained based on the approximation in each iteration. Considering comprehensively, the calculation process of Algorithm 1 is mainly divided into three stages: First of all, update the Hessian matrix of the Lagrangian function based on (24). Then, solve the quadratic programming sub-problem, and perform a constraint onedimensional search, thus we can obtain the maximum network capacity value in the end. Therefore, the complexity of each iteration of Algorithm 1 mainly comes from updating $\mathbf{H}_{k}$ based on $\mathbf{H}_{k-1}$ by 24 , solving a quadratic programming sub-problem, and performing the constraint one-dimensional search. To note, the BFGS correction method yields the complexity of $\mathrm{O}(M)$ with the output of $\mathbf{H}_{k}$. On the other hand, the method of using the BFGS correction formula to approximate the Hessian matrix of the objective function for solving a quadratic programming sub-problem can be regarded as a kind of quasi- Newton method. Considering the super-linear convergence of the quasi-Newton method, the complexity of solving the quadratic programming subproblem is $\mathrm{O}\left(M \ln \ln \left(\frac{1}{\varepsilon_{1}}\right)\right)$, where the termination criteria $\varepsilon_{1}$ determines the accuracy of solving the quadratic programming sub-problem. Finally the bisection method is applied with the complexity of $\mathrm{O}\left(M^{2} \log _{2}\left(\frac{1}{\varepsilon_{2}}\right)\right)$ [52], where the termination criteria $\varepsilon_{2}$ determines the accuracy of performing the constraint one-dimensional search. Therefore, Algorithm 1 yields the periteration complexity of $\mathrm{O}\left(M+M \ln \ln \left(\frac{1}{\varepsilon_{1}}\right)+M^{2} \log _{2}\left(\frac{1}{\varepsilon_{2}}\right)\right)$, which is of polynomial-time computational complexity.

\section{Performance Evaluation}

\section{A. Simulation Setup}

Based on the system model described in Section III-A. the entire train-ground communication system works in the $60 \mathrm{GHz} \mathrm{mm}$-wave frequency band. On the other hand, because mm-wave BSs are commonly used in small-scale and dense deployment scenarios, the simulation environment is set to be a square area with $500 \times 500 \mathrm{~m}^{2} .200$ users randomly appear in this area, and their position coordinates meet the uniform distribution of this area, and each user is associated with the nearest service device, i.e., the principle of "who is near, who serves." The track-side BS is located above the center of the area. Considering the actual operation scenario of the HSR, $\overline{\text { Algorithm } 1 \text { Quadratic Programming Algorithm based on }}$ Lagrangian Function in HSR Scenarios

1: Determine the locations of the BS and MRs;

2: Locate all users in this train-ground communication system and establishes the relationship between each user and the specific server;

3: Construct global network capacity function according to obtained communication system parameters;

4: Construct the Lagrangian function of the bandwidth allocation problem based on the objective function and constraints;

5: Determine the iteration starting point $\boldsymbol{\alpha}_{0}$ and the convergence accuracy $\sigma$;

6: Approximate the original bandwidth allocation optimization problem to a new quadratic programming subproblem at point $\boldsymbol{\alpha}_{0}$

7: Solve the obtained quadratic programming sub-problem, and let $\mathbf{S}_{0}=\mathbf{S}^{*}$;

8: Perform a constrained one-dimensional search on the global network capacity function in the direction $\mathbf{S}_{0}$ to obtain a new bandwidth allocation factor $\boldsymbol{\alpha}_{1}$;

9: $k=0$;

10: while $\boldsymbol{\alpha}_{k+1}$ does not meet the convergence accuracy do

11: $\quad k=k+1$;

12: Modify matrix $\mathbf{H}_{k}$ based on $\mathbf{H}_{k-1}$;

13: Simplify the original bandwidth allocation optimization problem into a new quadratic programming subproblem at point $\boldsymbol{\alpha}_{k}$;

14: Solve the obtained quadratic programming sub-problem and let $\mathbf{S}_{k}=\mathbf{S}^{*}$;

15: Perform a constrained one-dimensional search on the objective function of the original bandwidth allocation optimization problem in the direction $\mathbf{S}_{k}$, and then obtain the new bandwidth allocation factor $\boldsymbol{\alpha}_{k+1}$;

16: end while

17: Output optimal bandwidth allocation factor $\boldsymbol{\alpha}^{*}=\boldsymbol{\alpha}_{k+1}$;

18: Output Maximum network capacity value $f^{*}=f\left(\mathbf{X}_{k+1}\right)$;

the 9 MRs are arranged horizontally below the center of the area. The transmit power of the BS and MRs are $P_{t}$.

For mm-wave communications, we use the widely used real directional antenna model from IEEE 802.15.3c [9]. The model includes a linearly scaled Gaussian main lobe and a constant-level side lobe. Based on this model, the gain of a directional antenna in units of $\mathrm{dB}$ can be expressed as

$$
G(\theta)= \begin{cases}G_{0}-3.01 \cdot\left(\frac{2 \theta}{\theta_{-3 d B}}\right)^{2}, & 0^{\circ} \leq \theta \leq \frac{\theta_{m l}}{2} \\ G_{s l}, & \frac{\theta_{m l}}{2} \leq \theta \leq 180^{\circ}\end{cases}
$$

where $\theta \in\left[0^{\circ}, 180^{\circ}\right], G_{0}$ is the maximum antenna gain, which is given by $G_{0}=10 \log _{10}\left(\frac{1.6162}{\sin \left(\theta_{-3 d B} / 2\right)}\right)^{2}, \theta_{-3 d B}$ represents the half-power beamwidth, and $\theta_{m l}$ is the main lobe beamwidth in degrees. The relationship between $\theta_{m l}$ and $\theta_{-3 d B}$ can be expressed as $\theta_{m l}=2.6 \cdot \theta_{-3 d B}$. The sidelobe gain $G_{s l}$ is given by $G_{s l}=-0.4111 \cdot \ln \left(\theta_{-3 d B}\right)-10.579$. Other simulations parameters are given in Table $\mathrm{I}$ 
TABLE I

SiMULATION PARAMETERS

\begin{tabular}{lcc}
\hline Parameter & Symbol & Value \\
\hline Transmit power & $P_{t}$ & $1000 \mathrm{~mW}$ \\
Path loss exponent & $n$ & 2 \\
Transceiver efficiency factor & $\eta$ & 0.5 \\
Background noise & $N_{0}$ & $-134 \mathrm{dBm} / \mathrm{MHz}$ \\
Half-power beamwidth & $\theta_{-3 d B}$ & $30^{\circ}$ \\
Link blockage probability on average & $P_{b}$ & 0.2 \\
\hline
\end{tabular}

In order to evaluate the performance of the proposed SQP algorithm, the following four algorithms are chosen as baseline schemes:

1) PNOU (Priority based on the number of users): Considering the established communication scenario, the location of each user meets a random and uniform distribution in the communication system area. According to the "shortest distance criterion," when the association relationship between the user and the track-side BS or the MR is determined in advance, the BS and MRs can count the number of users associated with themselves, and use the number of associated users as the criterion for how much bandwidth is allocated. BS or MRs with a larger number of associated users will be allocated with relatively more bandwidth resources.

2) PD (Priority based on distance): After determining the association relationship between each user and the BS or MR according to the "shortest distance criterion," the BS and the MRs can collect the location information of their respective associated users, save the distance from each associated user, and use the average associated users' distance as the criterion for how much bandwidth is allocated. BS or MRs with a smaller average associated users' distance will be allocated relatively more bandwidth resources.

3) IP (Interior point algorithm): The interior point algorithm transforms the original constrained optimization problem into an unconstrained optimization problem. When constructing the objective function of the equivalent unconstrained optimization problem, it defines the objective function as a penalty function in the feasible region. It then solves the extreme point of penalty function in the feasible region. That is, the interior point algorithm ensures that the iteration point selected when solving the equivalent unconstrained optimization problem is always within the feasible region, as indicated by "interior point." When the selected iteration point moves from the feasible region to the constrained boundary, the value of the penalty function will increase sharply to infinity, which acts as a penalty to ensure that the iterative point will never touch the constrained boundary, so as to continuously approach the optimal solution of the original constrained optimization problem within the feasible region.

4) TR (Trust region algorithm): The fundamental idea of the trust region (TR) algorithm is a little different from that of the SQP algorithm. The SQP algorithm follows the principle of "adjusting the search direction and performing one-dimensional search." The TR algorithm, on the other hand, hopes to find the optimal solution of the problem in a domain. The "trust region" generally refers to a small neighborhood of the current iteration point. In this neighborhood, a sub-problem of the original nonlinear programming problem is solved to obtain the trial step size $S^{k}$. Then an evaluation function is used to determine whether to accept the trial step size and to determine the trust region within which the next round of iterative calculation will be done. At this time, if accept, then $\mathbf{X}_{k+1}=\mathbf{X}_{k}+\mathbf{S}_{k}$; otherwise $\mathbf{X}_{k+1}=\mathbf{X}_{k}$. The size of the new trust region is determined according to the quality of the trial step. If the trial step is good, the trust region will expand or remain unchanged in the next round of iterative calculation; otherwise, the trust region will decrease. It is worth noting that some key mathematical steps of the TR algorithm are consistent with the SQP algorithm, but the fundamental ideas of the two are different. In many iterative calculations, the TR algorithm keeps updating the search radius, while the SQP algorithm keeps updating the search direction.

Among the four comparison schemes listed above, the first two are greedy algorithms, i.e., they do not consider the overall optimality and always use a macro strategy based on a certain fixed idea, which is easier to implement. The latter two are common optimization algorithms for solving nonlinear programming problems. Their fundamental ideas have certain similarities with that of the SQP algorithm. They are also gradually approaching the optimal solution through iterations, but follow different specific guidelines in the search.

According to the optimization goal of problem (P1), the network capacity of the entire train-ground communication system, i.e., the sum of the average user's transmission rate of the BS and the average users' transmission rate of all MRs is used as performance metric, which is the optimization goal of this research.

$$
\max \bar{R}_{B S}+\sum_{i=1}^{\text {Rnum }} \bar{R}_{i}
$$

We will simulate the performance of the algorithms under different total bandwidth $W$ and different FD SI cancellation levels, i.e., $\beta$, at the MRs.

\section{B. Simulation Results and Discussions}

1) Under Different Total Bandwidth W: In this simulation, we apply the two greedy algorithms and the proposed SQP algorithm to evaluate the changes in network capacity as the system bandwidth $W$ is increased. The simulation results are shown in Fig. 5. For more rigorous, we display the data obtained by this simulation in Table [I] In this simulation, the SI cancellation capability of all FD MRs is set to $10^{-7}$.

It can be seen from Fig. 5 that as the total amount of bandwidth is increased, the three algorithms all exhibit a trend of linear growth. The performance of the proposed SQP algorithm is significantly better than that of PNOU and PD. Furthermore, as the system bandwidth is increased, the 


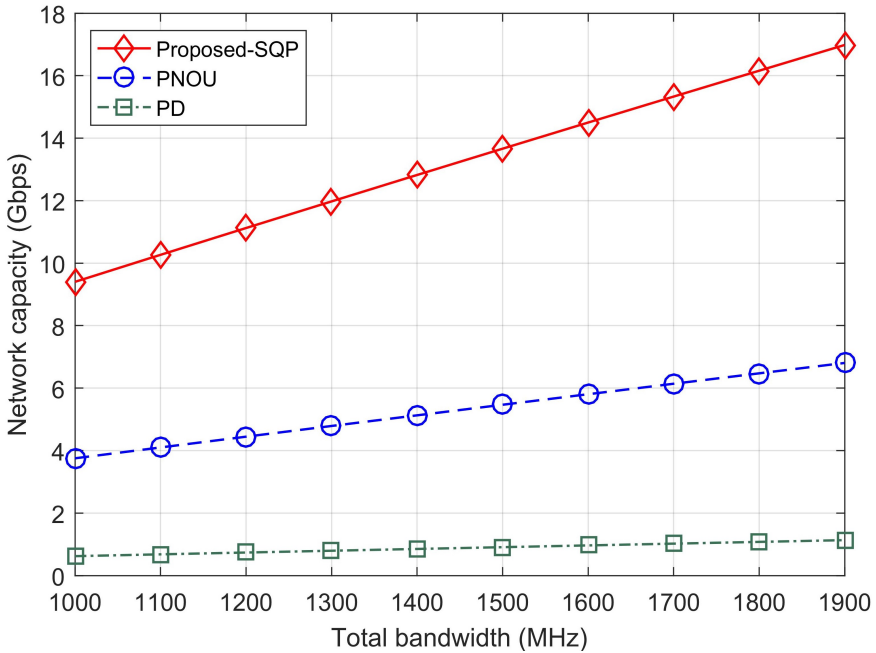

Fig. 5. Network capacity under different total bandwidth $W$.

TABLE II

NETWORK CAPACITY UNDER DIFFERENT TOTAL BANDWIDTH $W$

\begin{tabular}{c|c|c|c}
\hline Total bandwidth (MHz) & \multicolumn{3}{|c}{ Network capacity (Gbps) } \\
\hline & Proposed-SQP & PNOU & PD \\
\hline 1000 & 9.401 & 3.753 & 0.621 \\
1100 & 10.266 & 4.101 & 0.679 \\
1200 & 11.124 & 4.445 & 0.737 \\
1300 & 11.976 & 4.788 & 0.794 \\
1400 & 12.822 & 5.128 & 0.851 \\
1500 & 13.663 & 5.467 & 0.908 \\
1600 & 14.501 & 5.804 & 0.965 \\
1700 & 15.331 & 6.139 & 1.021 \\
1800 & 16.159 & 6.473 & 1.077 \\
1900 & 16.983 & 6.805 & 1.133 \\
\hline
\end{tabular}

network capacity achieved by the SQP algorithm increases at a faster rate than the two baseline schemes. With the proposed SQP algorithm, the sensitivity of the network capacity to the system bandwidth reaches about $9 \mathrm{Mbps} / 100 \mathrm{MHz}$, which is significantly higher than the $4.25 \mathrm{Mbps} / 100 \mathrm{MHz}$ achieved by the PNOU. With PD, the network capacity value does not increase significantly even as the total bandwidth is increased by $900 \mathrm{MHz}$. Especially, when the total bandwidth is $1200 \mathrm{MHz}$, the network capacity achieved by the SQP algorithm is about $11.2 \mathrm{Gbps}$, which is about 2.6 times of $4.4 \mathrm{Gbps}$ achieved by the PNOU, and about 12.4 times of $0.9 \mathrm{Gbps}$ achieved by the PD. When the total bandwidth is increased from $1200 \mathrm{MHz}$ to $1700 \mathrm{MHz}$, the network capacity achieved by the SQP algorithm increases by about $4.1 \mathrm{Gbps}$, which is about 2.4 times of $1.7 \mathrm{Gbps}$ achieved by the PNOU, and about 20.5 times of $0.2 \mathrm{Gbps}$ achieved by the PD.

As mentioned above, the user information collected by the global train-ground communication network is only each user's location and the respective distances to the server. The network performance of PD is the worst. This is because the BS and MRs are not evenly distributed in the established HSR scenario. According to the users' positions, except for some regional edge users, the $\mathrm{BS}$ is associated with users above the entire area. Therefore, users associated with the BS are more

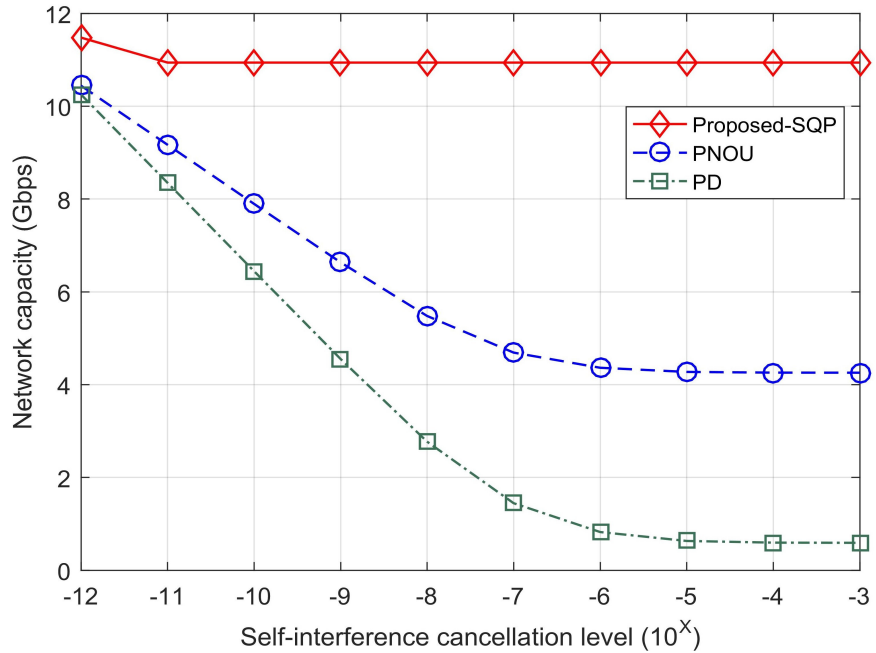

Fig. 6. Network capacity under different levels of SI cancellation.

TABLE III

NETWORK CAPACITY UNDER DIFFERENT LEVELS OF SI CANCELLATION

\begin{tabular}{c|c|c|c}
\hline \multicolumn{1}{c|}{$\mid$} & \multicolumn{3}{|c}{ Network capacity (Gbps) } \\
\hline & Proposed-SQP & PNOU & PD \\
\hline $1.0 \times 10^{-12}$ & 11.477 & 10.441 & 10.251 \\
$1.0 \times 10^{-11}$ & 10.943 & 9.168 & 8.349 \\
$1.0 \times 10^{-10}$ & 10.943 & 7.894 & 6.444 \\
$1.0 \times 10^{-9}$ & 10.943 & 6.635 & 4.552 \\
$1.0 \times 10^{-8}$ & 10.942 & 5.479 & 2.771 \\
$1.0 \times 10^{-7}$ & 10.942 & 4.691 & 1.444 \\
$1.0 \times 10^{-6}$ & 10.941 & 4.363 & 0.823 \\
$1.0 \times 10^{-5}$ & 10.941 & 4.274 & 0.631 \\
$1.0 \times 10^{-4}$ & 10.941 & 4.259 & 0.595 \\
$1.0 \times 10^{-3}$ & 10.941 & 4.257 & 0.591 \\
\hline
\end{tabular}

dispersed than that associated with the MRs. On the other hand, it is obvious that there are more users associated with the BS, and the PD algorithm allocates less bandwidth to the BS. This is why the average users' transmission rate of the BS cannot be effectively improved with PD.

2) Under Different Levels of SI Cancellation $\beta$ : Since the MRs adopt the FD communication mode, they also suffer residual SI. In order to study the performance of different algorithms in response to a sharp increase of SI, we also simulate the network capacity under different levels of SI cancellation. The results are presented in Fig. 6, and the specific experimental data are shown in Table III In this simulation, the initial system total bandwidth is fixed at $1200 \mathrm{MHz}$.

It can be seen from Fig. 6 that as the SI cancellation capability of the MRs is weakened, the network capacity achieved by the PNOU and PD both exhibit an exponential decline. When $\beta$ is increased above $10^{-5}$, the network capacity achieved by the PNOU and PD reaches the lowest value, which is about $4.2 \mathrm{Gbps}$ and $0.5 \mathrm{Gbps}$, respectively. Then they no longer decrease as $\beta$ is further increased.

In contrast, the network capacity achieved by the SQP algorithm remains at a relatively high level, as $\beta$ is increased. Especially, when $\beta$ is increased to $10^{-5}$, the network capacity 
TABLE IV

THE $\boldsymbol{\alpha}$ OBTAINED BY SQP WHEN THE SI CANCELLATION LEVEL IS $10^{-3}$

\begin{tabular}{c|c|c}
\hline$\alpha_{i}$ & Associated equipment & Value \\
\hline$\alpha_{0}$ & BS & 1.0000 \\
$\alpha_{1}$ & MR1 & $1.7598 \mathrm{e}-09$ \\
$\alpha_{2}$ & MR2 & $3.9526 \mathrm{e}-09$ \\
$\alpha_{3}$ & MR3 & $1.1182 \mathrm{e}-09$ \\
$\alpha_{4}$ & MR4 & $4.7802 \mathrm{e}-09$ \\
$\alpha_{5}$ & MR5 & $2.2247 \mathrm{e}-09$ \\
$\alpha_{6}$ & MR6 & $5.1305 \mathrm{e}-09$ \\
$\alpha_{7}$ & MR7 & $1.8028 \mathrm{e}-09$ \\
$\alpha_{8}$ & MR8 & $1.2927 \mathrm{e}-09$ \\
$\alpha_{9}$ & MR9 & $1.3782 \mathrm{e}-09$ \\
\hline
\end{tabular}

achieved by the SQP is about $11 \mathrm{Gbps}$, which is significantly better than $4.2 \mathrm{Gbps}$ of the PNOU and $0.5 \mathrm{Gbps}$ of the PD. When $\beta$ is increased from $10^{-12}$ to $10^{-5}$, the network capacity achieved by the SQP algorithm decreases by about $0.5 \mathrm{Gbps}$, which is about 0.08 times of $6.3 \mathrm{Gbps}$ achieved by the PNOU, and about 0.05 times of $9.7 \mathrm{Gbps}$ achieved by the PD. In other words, the proposed SQP algorithm has the ability to perceive the sudden increase of SI, and can coordinate the bandwidth resources of the entire network to ensure a consistently high network capacity. Considering that in the simulation, the SI cancellation level of each MR is the same, when the SI cancellation level drops significantly, the performance of the entire FD MR communication system drops sharply. We initially guessed that in order to ensure the network capacity of train-ground communication system, the SQP algorithm would make a decision to allocate almost all the bandwidth resources to the track-side BS operating in the HD mode. In addition, the number of users associated with the BS is relatively large, so the SQP algorithm still guarantees a high network capacity value under sharply increased $\beta$.

To verify this conjecture, the optimal bandwidth allocation factor $\boldsymbol{\alpha}$ computed by the SQP algorithm when the SI cancellation level is $10^{-3}$ is shown in Table IV It can be found that when the SI cancellation level of the MRs falls on the extremely poor order of magnitude, the bandwidth allocation decision obtained by the SQP algorithm is that the BS is assigned with almost all the bandwidth resources, which confirms the previous conjecture.

On the other hand, once the location of each user is determined in the train-ground communication system, the association relationship between a user is determined, and the bandwidth allocation factors obtained by PNOU and PD are determined. When the SI cancellation capability of the FD MRs is too weak, their received signals will be overwhelmed by the SI, and the user's transmission rate will be almost zero. The contribution of the average user's transmission rate through the MRs to the overall network capacity will be almost zero. This is the reason why the network capacity reaches its lowest under PNOU and PD when the SI cancellation capability of the MRs is too small. The lowest capacity mainly comes from the HD BS, because it is not limited by the SI cancellation capability of the MRs. When the level of SI cancellation changes, as long as the bandwidth resources allocated to the BS remain unchanged, the users' transmission
TABLE V

THE $\boldsymbol{\alpha}$ OBTAINED BY SQP WHEN THE SI CANCELlation LEVEL IS $10^{-9}$ AND $10^{-6}$

\begin{tabular}{c|c|c|c}
\hline$\alpha_{i}$ & Associated equipment & Value & Value \\
\hline & & $\beta=10^{-9}$ & $\beta=10^{-6}$ \\
\hline$\alpha_{0}$ & BS & 0.1921 & 0.3952 \\
$\alpha_{1}$ & MR1 & 0.0836 & 0.0161 \\
$\alpha_{2}$ & MR2 & 0.0344 & 0.0196 \\
$\alpha_{3}$ & MR3 & 0.0527 & 0.1159 \\
$\alpha_{4}$ & MR4 & 0.1504 & 0.1383 \\
$\alpha_{5}$ & MR5 & 0.0825 & 0.0306 \\
$\alpha_{6}$ & MR6 & 0.0881 & 0.0132 \\
$\alpha_{7}$ & MR7 & 0.1440 & 0.0146 \\
$\alpha_{8}$ & MR8 & 0.0899 & 0.1259 \\
$\alpha_{9}$ & MR9 & 0.0822 & 0.1306 \\
\hline
\end{tabular}

rates associated with the BS will not be affected.

With the above considerations and reviewing Fig. 6, we find that when the SI cancellation level at the FD MRs is between $10^{-11}$ and $10^{-3}$, the global network capacity is basically stable at about $11 \mathrm{Gbps}$. Does this mean that, when the SI cancellation level at the FD MRs drops to $10^{-11}$, the SQP algorithm directly makes the decision shown in Table IV] i.e., almost all the bandwidth resources are straightforwardly allocated to the BS? We run two more sets of simulations, which are also based on the simulation parameters shown in Table I] The difference between the two sets of simulations is that, the first set of simulations are carried out for $\beta=10^{-9}$, and the second set is for $\beta=10^{-6}$. The bandwidth allocation results achieved by the SQP algorihtm are presented in Table $\mathrm{V}$

From Table $\mathrm{V}$, it can be seen that when the SI cancellation level of the FD MRs deteriorates, the SQP algorithm does not allocate nearly all the bandwidth resources to the trackside BS. As the level of SI cancellation deteriorates, more bandwidth resources are gradually allocated to the BS. This decision is in line with the actual situation, i.e., although the level of SI cancellation at the MRs has deteriorated, if the SI has not reached the level of overwhelming desired signals, the normal communications of users associated with the MR should still be helpful. This helps maintain fairness between users associated with the BS and MRs. From this point of view, in Fig. 6, the network capacity achieved by the SQP algorithm is stabilized at $11 \mathrm{Gbps}$.

3) Comparison with Other Optimization Algorithms: We also used two other optimization algorithms for constrained nonlinear programming problems, i.e., IP and TR, as baseline schemes. In the simulations, we find that if other simulation parameters are the same, there is only a difference of less than $1 \mathrm{Kbps}$ between the network capacity achieved by the proposed SQP algorithm and that achieved by the IP and the TR algorithm. Since the network capacity of the train-ground communication system is on the order of Gbps, such a small difference is negligible. Therefore, network capacity under different system bandwidths and different SI cancellation levels obtained by the proposed SQP algorithm are close to that achieved by the IP and the TR algorithm. Instead, we compare the three optimization schemes with respect to their execution time and the relative value of the maximum network 


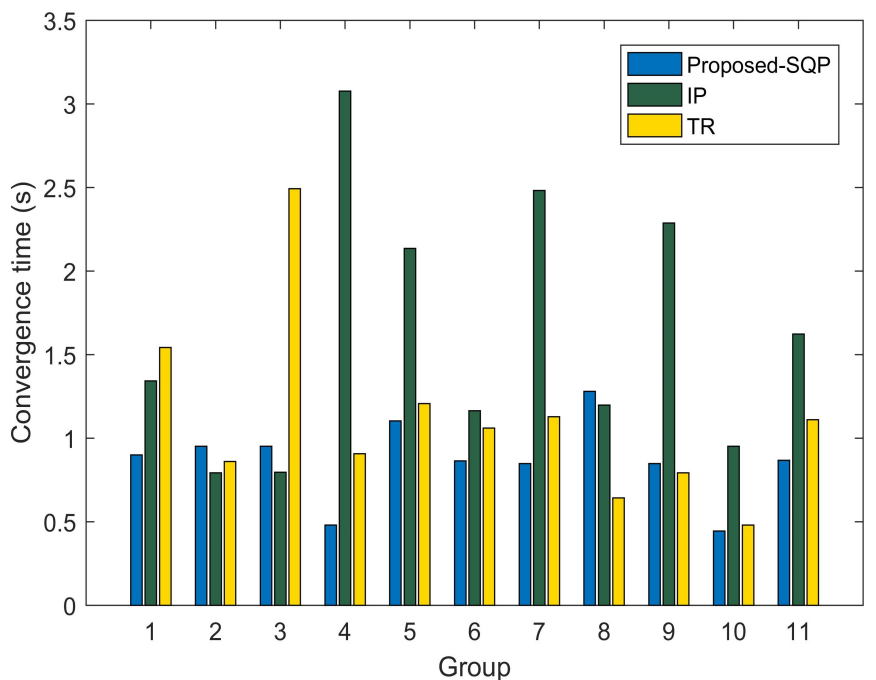

Fig. 7. Convergence time of the three optimization algorithms.

TABLE VI

CONVERGENCE TIME OF THE THREE OPTIMIZATION ALGORITHMS

\begin{tabular}{c|c|c|c}
\hline Group & \multicolumn{3}{|c}{ Convergence time (s) } \\
\hline & Proposed-SQP & IP & TR \\
\hline 1 & 0.901 & 1.343 & 1.542 \\
2 & 0.952 & 0.792 & 0.861 \\
3 & 0.952 & 0.796 & 2.492 \\
4 & 0.481 & 3.076 & 0.908 \\
5 & 1.104 & 2.136 & 1.208 \\
6 & 0.864 & 1.164 & 1.061 \\
7 & 0.848 & 2.248 & 1.128 \\
8 & 1.281 & 1.199 & 0.643 \\
9 & 0.848 & 2.288 & 0.792 \\
10 & 0.444 & 0.952 & 0.481 \\
11 & 0.867 & 1.623 & 1.111 \\
\hline
\end{tabular}

capacity obtained. The results are presented in Figs. 7 and 8 . where the 11th groups of data represent the average of the previous 10 groups of data, and and the specific experimental data are shown in Table VI and Table VII Note that the simulation parameters of each group in Figs. 7 and 8 are given in Table I; the total bandwidth resource is fixed at $1200 \mathrm{MHz}$; and the SI cancellation level of the MRs is fixed at $10^{-7}$. In the beginning of each group of experiments, the location and associated information of 200 users are randomly generated, and finally the average value of the 10 groups of experiments will be calculated and presented.

It should be noted that when using any iterative optimization algorithm to solve a nonlinear programming problem, it is necessary to set the parameter of accuracy requirement, and the iteration process stops when the result that satisfies the accuracy requirement is obtained. Therefore, for a specific optimization algorithm, increasing the required accuracy will inevitably lead to an increase in execution time. In the above 10 sets of experiments, the accuracy requirements of the three algorithms are set to the Kbps level in advance. This allows us to compare the convergence speed of the three algorithms.

First observe the average convergence time shown in Fig. 7 .

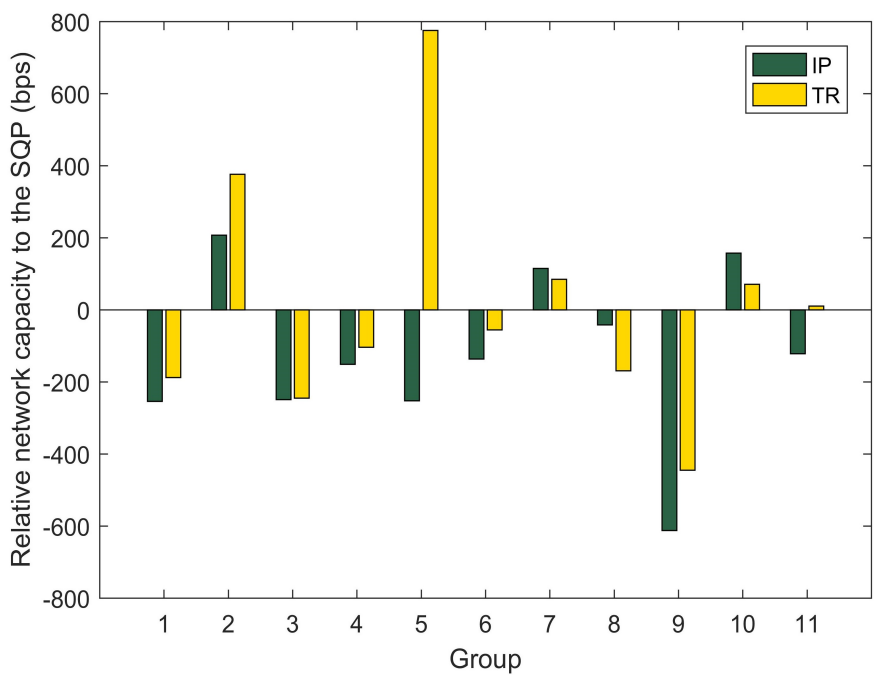

Fig. 8. Relative value of network capacity achieved by the three optimization algorithms.

TABLE VII

RELATIVE VALUE OF NETWORK CAPACITY ACHIEVED BY THE THREE OPTIMIZATION ALGORITHMS

\begin{tabular}{c|c|c}
\hline Group & \multicolumn{2}{|c}{ Relative network capacity to the SQP (bps) } \\
\hline & IP & TR \\
\hline 1 & 254.15 & 187.65 \\
2 & -207.34 & -376.03 \\
3 & 248.94 & 245.2 \\
4 & 151.03 & 103.74 \\
5 & 251.84 & -775.57 \\
6 & 136.11 & 55.243 \\
7 & -115.49 & -85.262 \\
8 & 41.915 & 168.69 \\
9 & 612.18 & 444.62 \\
10 & -157.51 & -71.381 \\
11 & 121.58 & -10.309 \\
\hline
\end{tabular}

From the point of view of execution time, the proposed SQP algorithm outperforms both IP and TR, and TR is faster than IP. Also the convergence time of the proposed SQP algorithm is the most stable one among the three algorithms. In Fig. 8, with the simulation parameters and convergence accuracy fixed, taking the data of the fifth group as an example, the maximum network capacity value calculated by the SQP algorithm is $0.252 \mathrm{Kbps}$ higher than that calculated by IP, and $0.776 \mathrm{Kbps}$ lower than that calculated by TR. From the calculated average relative value of the maximum network capacity shown in Fig. 8, the maximum network capacity value obtained by the SQP algorithm is $0.122 \mathrm{Kbps}$ lower than that obtained by IP, and $0.01 \mathrm{Kbps}$ larger than that obtained by TR. The IP algorithm has the longest average running time, but also with a slightly better network capacity. It may not worth pursuing for such a small gain in capacity at the greatly extended execution time. The average running time of the TR algorithm is in the middle of the three algorithms, but the average network capacity value obtained by TR is lower than that of the SQP algorithm.

On the other hand, fewer stations are set along the high- 
speed railways, thus ensuring that the high-speed trains can maintain a long-term high-speed and stable operation. When the train is running at a high and stable speed, the moving line is deterministic and the train's position is predictable, so the power of the received signal is also highly predictable. This feature makes it easier to optimize the management of the mobility and resources of the communication system in HSR scenarios. In the investigated $\mathrm{mm}$-wave train-ground communication system, if the train's position can be predicted, the relative distance between MRs and users can be determined, and the bandwidth allocation optimization problem can be formulated for a period of time in the future, and the proposed algorithm can also make decisions in advance.

In the above simulations, we also find an interesting phenomenon: When the simulation parameters are unchanged and the SQP algorithm is run multiple times to solve the P1, the maximum network capacity value obtained within the convergence accuracy corresponds to a variety of optimal bandwidth allocation factors. The values in these optimal bandwidth allocation factors are slightly different, but the maximum network capacity values obtained by using them are the same. This also explains that problem (P1) has multiple local optimal solutions, and there are several local optimal solutions that are within the convergence accuracy of the global optimal solution, so they can all be directly regarded as the global optimal solution. From the basic idea of the SQP algorithm, this means that there are many search directions for the global optimal solution, which greatly accelerates the convergence speed, reduces the number of algorithm iterations, and improves the performance of the proposed SQP algorithm.

\section{Conclusions}

In this paper, we introduced a SQP algorithm based on the Lagrangian function to solve the problem of bandwidth allocation between the track-side BS and FD MRs in a trainground communication system. The SQP algorithm ensures that the optimal solution of the problem can be quickly approached, and the Lagrangian function ensures that the sub-problems of the original problem approximated in each iteration are convex, i.e., the local optimal solution of the problem is the global optimal solution. Extensive simulation results demonstrated that the proposed SQP algorithm can effectively improve the network capacity of the train-ground communication in HSR scenarios while maintaining a certain anti-SI ability, when compared with four baseline schemes. For the future work, we will deeply investigate the blockage problem in the studied $\mathrm{mm}$-wave train-ground communication system, and consider using IRS to propose a solution.

\section{REFERENCES}

[1] B. Ai, A. F. Molisch, M. Rupp and Z. Zhong, "5G key technologies for smart railways," in Proceedings of the IEEE, vol. 108, no. 6, pp. 856-893, June 2020.

[2] D. Fan, Z. Zhong, G. Wang, and F. Gao, "Doppler shift estimation for high-speed railway wireless communication systems with largescale linear antennas," in Proc. Int. Workshop High Mobility Wireless Commun., pp. 96-100, Oct. 2015.

[3] M. Gao et al., "Dynamic mmWave beam tracking for high speed railway communications," in Proc. IEEE Wireless Commun. Netw. Conf. Workshops (WCNCW), pp. 278-283, Apr. 2018.
[4] B. Lannoo, D. Colle, M. Pickavet, and P. Demeester, "Radio-over-fiberbased solution to provide broadband Internet access to train passengers," IEEE Communications Magazine, vol. 45, no. 2, pp. 56-62, Feb. 2007.

[5] M. Elkashlan, T. Q. Duong, and H. H. Chen, "Millimeter-wave communications for 5G: fundamentals: Part I [Guest Editorial]," IEEE Communications Magazine, vol. 52, no. 9, pp. 52-54, Sept. 2014.

[6] Z. Zhang, X. Chai, K. Long, A. V. Vasilakos and L. Hanzo, "Full duplex techniques for 5G networks: self-interference cancellation, protocol design, and relay selection," IEEE Communications Magazine, vol. 53, no. 5, pp. 128-137, May 2015.

[7] C. H. Doan, S. Emami, D. A. Sobel, A. M. Niknejad, and R. W. Brodersen, "Design considerations for $60 \mathrm{GHz}$ CMOS radios," IEEE Communications Magazine, vol. 42, no. 12, pp. 132-140, Dec. 2004.

[8] ECMC TC48, "ECMA standard 387-High rate $60 \mathrm{GHz}$ PHY, MAC and HDMI PAL," 2nd edition Dec. 2010.

[9] IEEE 80215 WPAN Millimeter Wave Alternative PHY Task Group 3c (TG3c), "Wireless Medium Access Control (MAC) and Physical Layer (PHY) specifications for high rate Wireless Personal Area Networks (WPANs) (Amendement 2: Millimeter-wave-based Alternative Physical Layer Extension)," Oct. 2009.

[10] IEEE 80211ad Standard, "Wireless LAN Medium Access Control (MAC) and Physical Layer(PHY) Specifications (Amendment 3: Enhancements for Very High Throughput in the $60 \mathrm{GHz}$ Band)," Dec. 2012.

[11] R. W. Heath, N. Gonzlez-Prelcic, S. Rangan, W. Roh and A. M. Sayeed, "An overview of signal processing techniques for millimeter wave MIMO systems," IEEE Journal of Selected Topics in Signal Processing, vol. 10, no. 3, pp. 436-453, April 2016.

[12] R. Mudumbai, S. K. Singh, and U. Madhow, "Medium access control for $60 \mathrm{GHz}$ outdoor mesh networks with highly directional links," in Proc. IEEE INFOCOM 2009 (Mini Conference), Rio de Janeiro, Brazil, pp. 2871-2875, Apr. 2009.

[13] A. Sadeghi, M. Luvisotto, F. Lahouti, S. Vitturi, and M. Zorzi, "Statistical QoS analysis of full duplex and half duplex heterogeneous cellular networks," in Proc. IEEE ICC 2016, Kuala Lumpur, Malaysia, pp. 1-6, May 2016.

[14] D. P. Bertsekas, Constrained Optimization and Lagrange Multiplier Methods, Cambridge, MA: Academic Press, 1982.

[15] I. K. Son, S. Mao, M. X. Gong, and Y. Li, "On frame-based scheduling for directional mmWave WPANs," in Proc. IEEE INFOCOM 2012, Orlando, FL, pp. 2149-2157, Mar. 2012.

[16] I. K. Son, S. Mao, Y. Li, M. Chen, M.X. Gong, and T.S. Rappaport, "Frame-based medium access control for 5G wireless networks," Springer MONET J., vol. 20, no. 6, pp. 763-772, Dec. 2015.

[17] M. Jain, J. I. Choi, T. Kim, D. Bharadia, S. Seth, K. Srinivasan, P. Levis, S. Katti, and P. Sinha, "Practical, real-time, full duplex wireless," in Proc. ACM MobiCom 2011, Las Vegas, NV, pp. 301-312, Sept. 2011.

[18] H. Cui, M. Ma, L. Song and B. Jiao, "Relay selection for two-way full duplex relay networks With amplify-and-forward protocol," IEEE Transactions on Wireless Communications, vol. 13, no. 7, pp. 37683777, July 2014.

[19] K. M. Rahman, N. Hakem and B. Barua, "FD-MIMO relay selfinterference cancellation using space projection algorithms," 2017 IEEE 9th Latin-American Conference on Communications (LATINCOM), Guatemala City, pp. 1-5, 2017.

[20] Y. Niu, Y. Li, D. Jin, L. Su, A. V. Vasilakos, "A Survey of millimeter wave communications (mmWave) for 5G: Opportunities and challenges," Wireless Networks, vol. 21, no. 8, pp. 2657-2676, Nov. 2015.

[21] Y. Niu, C. Gao, Y. Li, L. Su, D. Jin, Y. Zhu, and D. O. Wu, "Energy-efficient scheduling for mmwave backhauling of small cells in heterogeneous cellular networks," IEEE Transactions on Vehicular Technology, vol. 66, no. 3, pp. 2674-2687, Mar. 2017.

[22] Y. Chen, B. Ai, Y. Niu, K. Guan, and Z. Han, "Resource allocation for device-to-device communications underlaying heterogeneous cellular networks using coalitional games," IEEE Transactions on Wireless Communications, vol. 17, no. 6, pp. 4163-4176, June 2018.

[23] L. Wang, B. Ai, Y. Niu, X. Chen, and P. Hui, "Energy-efficient power control of train-ground mmWave communication for high-speed trains," IEEE Transactions on Vehicular Technology, vol. 68, no. 8, pp. 77047714, Aug. 2019.

[24] D. Z. Wen and G. D. Yu, "Time-division cellular networks with fullduplex base stations," IEEE Communications Letters, vol. 20, no. 2, pp. 392-395, Feb. 2016.

[25] W. G. Ding, Y. Niu, H. Wu, Y. Li, and Z. D. Zhong, "QoS-aware full-duplex concurrent scheduling for millimeter wave wireless backhaul networks," IEEE Access J., vol. 6, pp. 25313-25322, May 2018. 
[26] C. Skouroumounis, C. Psomas, and I. Krikidis, "Heterogeneous FD-mmWave cellular networks with cell center/edge users," IEEE Transactions on Communications, vol. 67, no. 1, pp. 791-806, Jan. 2019.

[27] Y. Wang, Y. Niu, H. Wu, Z. Han, B. Ai, and Q. Wang, "Sub-channel allocation for device-to-device underlaying full-duplex mmWave small cells using coalition formation games," IEEE Transactions on Vehicular Technology, vol. 68, no. 12, pp. 11915-11927, Dec. 2019.

[28] S. Xiao, X. Zhou, Y. Yuan-Wu, G. Y. Li, and W. Guo, "Robust resource allocation in full-duplex-enabled OFDMA femtocell networks," IEEE Transactions on Wireless Communications, vol. 16, no. 10, pp. 63826394, Oct. 2017.

[29] D. Liu, H. Zhang, W. Zheng, and X. Wen, "The sub-channel allocation algorithm in femtocell networks based on Ant Colony Optimization," in Proc. IEEE MILCOM 2012, Orlando, FL, pp. 1-6, Oct. 2012.

[30] Y. Zhu, Y. Niu, J. Li, D. O. Wu, Y. Li, and D. Jin, "QoS-aware scheduling for small cell millimeter wave mesh backhaul," in Proc. IEEE ICC 2016, Kuala Lumpur, Malaysia, pp. 1-6, May 2016.

[31] M. Gao et al., "Efficient hybrid beamforming with anti-blockage design for high-speed railway communications," IEEE Transactions on Vehicular Technology, vol. 69, no. 9, pp. 9643-9655, Sept. 2020.

[32] S. Kutty and D. Sen, "Beamforming for millimeter wave communications: An inclusive survey," IEEE Communications Surveys and Tutorials, vol. 18, no. 2, pp. 949-973, Secondquarter 2016.

[33] G. Yang, J. Du and M. Xiao, "Maximum throughput path selection with random blockage for indoor $60 \mathrm{GHz}$ relay networks," IEEE Transactions on Communications, vol. 63, no. 10, pp. 3511-3524, Oct. 2015.

[34] V. Va, X. Zhang and R. W. Heath, "Beam Switching for Millimeter Wave Communication to Support High Speed Trains," 2015 IEEE 82nd Vehicular Technology Conference (VTC2015-Fall), Boston, MA, USA, pp. 1-5, 2015.

[35] L. Yan, X. Fang, L. Hao and Y. Fang, "A Fast Beam Alignment Scheme for Dual-Band HSR Wireless Networks," in IEEE Transactions on Vehicular Technology, vol. 69, no. 4, pp. 3968-3979, April 2020.

[36] Y. Li, J. G. Andrews, F. Baccelli, T. D. Novlan and C. J. Zhang, "Design and Analysis of Initial Access in Millimeter Wave Cellular Networks," in IEEE Transactions on Wireless Communications, vol. 16, no. 10, pp 6409-6425, Oct. 2017.

[37] 3GPP TR 38.804 V14.0.0, Technical specification group radio access network; Study on new radio access technology; Radio interface protocol aspects (Release 14), Mar. 2017.

[38] E. Dahlman, S. Parkvall, and J. Skold, LTE/LTE-Advanced for Mobile Broadband. Elsevier Ltd, 2011

[39] Y. Wang, S. Mao, and T.S. Rappaport, "On directional neighbor discovery in mmWave networks," in Proc. IEEE ICDCS 2017, Atlanta, GA, pp.1704-1713, June 2017.

[40] Z. He, S. Mao, and T.S. Rappaport, "On link scheduling under blockage and interference in $60 \mathrm{GHz}$ ad hoc networks," IEEE Access Journal, vol.3, pp.1437-1449, Sept. 2015.

[41] J. Yang, B. Ai, D. He, L.Wang, Z. Zhong, and A. Hrovat, "A simplified multipath component modeling approach for high-speed train channel based on ray tracing," Wireless Commun. Mobile Comput., vol. 2017, no. 333 , pp. $1-14$, Oct. 2017

[42] D. He et al., "Channel measurement, simulation, and analysis for highspeed railway communications in $5 \mathrm{G}$ millimeter-wave band," IEEE Transactions on Intelligent Transportation Systems, vol. 19, no. 10, pp. 3144-3158, Oct. 2018.

[43] T. Kawanishi et al., "Proposal of a new working document of a draft new apt report on millimeter-wave band railway radiocommunication systems between train and trackside and its work plan," in Proc. 20th Meeting APT Wireless Group, pp. 1-12, 2016.

[44] M. Gao et al., "Edge caching and content delivery with minimized delay for both high-speed train and local users," 2019 IEEE Global Communications Conference (GLOBECOM), Waikoloa, HI, USA, pp. $1-6,2019$.

[45] M. Duarte, A. Sabharwal, V. Aggarwal, R. Jana, K. K. Ramakrishnan, C. Rice, and N. K. Shankaranayanan, "Design and characterization of a full-duplex multi-antenna system for WiFi networks," IEEE Transactions on Vehicular Technology, vol. 63, no. 3, pp. 1160-1177, Mar. 2014.

[46] Z. Jiang and S. Mao, "Energy delay trade-off in multi-channel fullduplex wireless LANs," IEEE Internet of Things Jounal, vol.4, no.3, pp.658-669, June 2017.

[47] Y. Wang and S. Mao, "On distributed power control in full duplex wireless networks," Elsevier Digital Communications and Networks Journal, vol.3, no.1 pp.1-10, Feb. 2017.

[48] M. Feng, S. Mao, and T. Jiang, "Joint duplex mode selection, channel allocation, and power control for full-duplex cognitive femtocell net- works," Elsevier Digital Communications and Networks Journal, vol.1, no.1, pp.30-44, Feb. 2015.

[49] Z. Y. Xiao, P. F. Xia, and X. G. Xia, "Full-duplex millimeter-wave communication," IEEE Wireless Communications Magazine, vol. 24, no. 6, pp. 136-143, Dec. 2017

[50] P. Anokye, R. K. Ahiadormey, C. Song, and K. J. Lee, "Achievable sum-rate analysis of massive MIMO full-duplex wireless backhaul links in heterogeneous cellular networks," IEEE Access J., vol. 6, pp. 2345623469, May. 2018.

[51] U. M. Garcia Palomares and O. L. Mangasarian, "Superlinearly convergent quasi-newton algorithms for nonlinearly constrained optimization problems," Mathematical Programming, vol.11, pp.1-13, Dec. 1976.

[52] S. Boyd and L. Vandenberghe, Convex Optimization. Cambridge, U.K.: Cambridge Univ. Press, 2004. 